\title{
Positive Periodic Solutions of Third-Order Ordinary Differential Equations with Delays
}

\author{
Yongxiang $L i$ and Qiang $L i$ \\ Department of Mathematics, Northwest Normal University, Lanzhou 730070, China \\ Correspondence should be addressed to Yongxiang Li; liyxnwnu@163.com
}

Received 24 October 2013; Accepted 30 December 2013; Published 20 February 2014

Academic Editor: Zhanbing Bai

Copyright (C) 2014 Y. Li and Q. Li. This is an open access article distributed under the Creative Commons Attribution License, which permits unrestricted use, distribution, and reproduction in any medium, provided the original work is properly cited.

The existence results of positive $\omega$-periodic solutions are obtained for the third-order ordinary differential equation with delays $u^{\prime \prime \prime}(t)+a(t) u(t)=f\left(t, u\left(t-\tau_{0}\right), u^{\prime}\left(t-\tau_{1}\right), u^{\prime \prime}\left(t-\tau_{2}\right)\right), t \in \mathbb{R}$, where $a \in C(\mathbb{R},(0, \infty))$ is $\omega$-periodic function and $f: \mathbb{R} \times[0, \infty) \times \mathbb{R}^{2} \rightarrow$ $[0, \infty)$ is a continuous function which is $\omega$-periodic in $t$, and $\tau_{0}, \tau_{1}, \tau_{2}$ are positive constants. The discussion is based on the fixedpoint index theory in cones.

\section{Introduction}

In this paper, we discuss the existence of positive $\omega$-periodic solutions for the third-order ordinary differential equation with delays

$$
\begin{array}{r}
u^{\prime \prime \prime}(t)+a(t) u(t)=f\left(t, u\left(t-\tau_{0}\right), u^{\prime}\left(t-\tau_{1}\right), u^{\prime \prime}\left(t-\tau_{2}\right)\right), \\
t \in \mathbb{R},
\end{array}
$$

where $a \in C(\mathbb{R},(0, \infty))$ is a $\omega$-periodic function, $f$ : $\mathbb{R} \times[0, \infty) \times \mathbb{R}^{2} \rightarrow[0, \infty)$ is a continuous function, and $f(t, x, y, z)$ is $\omega$-periodic in $t$, and $\omega, \tau_{0}, \tau_{1}, \tau_{2}$ are positive constants.

In recent years, the existence of periodic solutions for first-order and second-order delay differential equations has been researched by many authors; see [1-5] for the firstorder equations and see [6-12] for the second-order ones. In some practice models, only positive periodic solutions are significant. In $[3,8,9,11,12]$, the authors obtained the existence of positive periodic solutions for some first-order and second-order delay differential equations by using Krasnoselskii's fixed-point theorem of cone mapping. But, few people consider the existence of positive periodic solutions for third-order delay differential equations.

The third-order delay differential equations have their important physical contexts, for example, which can be formulated from the problem of the wave solution of the Korteweg-de Vries (KdV) equation with time delay. Recently, Zhao and $\mathrm{Xu}$ [13] pointed out that the KdV equation with time delay has more actual significance and they considered the solitary wave solution of the following $\mathrm{KdV}$ equation with time delay:

$$
\begin{aligned}
U_{t}(x, t) & +U(x, t-\tau) U_{x}(x, t)+\tau U_{x x}(x, t-\tau) \\
& -U_{x x x}(x, t)=0,
\end{aligned}
$$

where $\tau$ is a given constant and $U_{x x}(x, t-\tau)$ means the backward diffusion with time delay. They looked for a wave solution $U(x, t)=\varphi(x+c t)$ with $c>0$ and from (2) obtained the following third-order delay ordinary differential equation of the profile $\varphi$ :

$$
c \varphi^{\prime}(\xi)+\varphi(\xi-c \tau)+\tau \varphi^{\prime \prime}(\xi-c \tau)-\varphi^{\prime \prime \prime}(\xi)=0, \quad \xi \in \mathbb{R} .
$$

Equation (3) is a special form of (1). If we look for a periodic wave solution of (2), we need to discuss the existence of the periodic solution of the delay ordinary differential equation (3). Hence, the existence problem of periodic solutions of the general third-order delay differential equation (1) is a significant topic.

For the third-order ordinary differential equations without delays, the existence of periodic solutions has been considered by several authors; see [14-23] and references therein. 
Some theorems and methods of nonlinear functional analysis have been applied to research on this problem, such as the methods of topological degree and Leray-Schauder fixedpoint theorem $[14,19]$, the upper and lower solutions method and monotone iterative technique [15-17], the implicit function theorem [18], and Mawhin coincidence degree theory [20]. Especially, in recent years, the fixed-point theorem of Krasnoselskii's cone expansion or compression type has been availably applied to some special third-order periodic boundary problems of ordinary differential equations, and some results of existence and multiplicity of positive periodic solutions have been obtained; see [21, 22]. In [21], Chu and Zhou considered the periodic boundary value problem for the third-order equation

$$
u^{\prime \prime \prime}(t)+\rho^{3} u(t)=f(t, u(t)), \quad t \in[0,2 \pi],
$$

where $\rho \in(0,1 / \sqrt{3})$ is a constant and $f \in C([0,2 \pi] \times(0, \infty))$. Using the Krasnoselskii's fixed-point theorem in cones, they obtained the existence results of positive solutions. Their results extended the one obtained by the Schauder fixedpoint theorem in [19]. In [22], by the Krasnoselskii's fixedpoint theorem in cones, Feng established some existence and multiplicity results of positive periodic solutions for the thirdorder equation

$$
u^{\prime \prime \prime}(t)+\alpha u^{\prime \prime}(t)+\beta u^{\prime}(t)=f(t, u(t)), \quad t \in[0,2 \pi],
$$

where $\alpha$ and $\beta$ are positive constants and satisfy certain conditions. In [23], the present author extended and improved the results in $[9,10]$ to the general third-order equation

$$
u^{\prime \prime \prime}(t)=f\left(t, u(t), u^{\prime}(t), u^{\prime \prime}(t)\right), \quad t \in \mathbb{R}
$$

that nonlinearity $f$ explicitly contains derivative terms $u^{\prime}(t)$ and $u^{\prime \prime}(t)$. However, all of these works are on the third-order equations without delays and the argument methods are not applicable to the delay equation (1).

Motivated by the facts mentioned above, we research the existence of positive periodic solutions of the third-order delay equation (1). We will use the fixed-point index theory in cones in a meticulous way to obtain the essential conditions on the existence of positive periodic solutions of (1). Our main results will be given in Section 3. Some preliminaries to discuss (1) are presented in Section 2.

\section{Preliminaries}

Let $C_{\omega}(\mathbb{R})$ denote the Banach space of all continuous $\omega$ periodic function $u(t)$ with norm $\|u\|_{C}=\max _{0 \leq t \leq \omega}|u(t)|$. Generally, for $n \in \mathbb{N}$, we use $C_{\omega}^{n}(\mathbb{R})$ to denote the Banach space of all $n$ th-order continuous differentiable $\omega$-periodic function with the norm $\|u\|_{C^{n}}=\sum_{k=0}^{n}\left\|u^{(k)}\right\|_{C}$. Let $C_{\omega}^{+}(\mathbb{R})$ denote the cone of all nonnegative functions in $C_{\omega}(\mathbb{R})$.

Let $M>0$ be a constant. For $h \in C_{\omega}(\mathbb{R})$, we consider the existence of $\omega$-periodic solution of the linear third-order differential equation

$$
u^{\prime \prime \prime}(t)+M u(t)=h(t), \quad t \in \mathbb{R} .
$$

It is easy to verify that the linear third-order boundary value problem

$$
\begin{gathered}
u^{\prime \prime \prime}(t)+M u(t)=0, \quad t \in[0, \omega], \\
u(0)-u(\omega)=0, \quad u^{\prime}(0)-u^{\prime}(\omega)=0, \\
u^{\prime \prime}(0)-u^{\prime \prime}(\omega)=1
\end{gathered}
$$

has a unique solution. We denote the solution by $\Phi(t)$. By [16, Lemma 2.1], the $\omega$-periodic solution of (7) can be expressed by $\Phi$. By [16, Lemma 2.1] or a direct calculation, we easily obtain the following lemma.

Lemma 1. Let $M>0$. Then, for every $h \in C_{\omega}(\mathbb{R})$, the linear equation (7) has a unique w-periodic solution $u(t)$ which is given by

$$
u(t)=\int_{t-\omega}^{t} \Phi(t-s) h(s) d s:=P h(t), \quad t \in \mathbb{R} .
$$

Moreover, $P: C_{\omega}(\mathbb{R}) \rightarrow C_{\omega}^{2}(\mathbb{R})$ is a completely continuous linear operator.

Lemma 2. Let $0<M<(2 \pi / \sqrt{3} \omega)^{3}$. Then, the solution $\Phi$ of the linear third-order boundary value $(8)$ is positive on $[0, \omega]$.

Proof. Let $\rho=\sqrt[3]{M}$. It is easy to prove that the linear secondorder boundary value problem

$$
\begin{aligned}
& u^{\prime \prime}(t)-\rho u^{\prime}(t)+\rho^{2} u(t)=0, \quad t \in[0, \omega], \\
& u(0)-u(\omega)=0, \quad u^{\prime}(0)-u^{\prime}(\omega)=1
\end{aligned}
$$

has a unique solution $\Phi_{2}(t)$ which is given by

$$
\begin{aligned}
& \Phi_{2}(t) \\
& =\frac{e^{(\rho / 2) t}\left(e^{\rho \omega / 2} \sin (\sqrt{3} \rho / 2)(\omega-t)+\sin (\sqrt{3} \rho / 2) t\right)}{(\sqrt{3} \rho / 2)\left(\left(e^{\rho \omega / 2}-1\right)^{2}+2 e^{\rho \omega / 2}(1-\cos (\sqrt{3} \rho / 2) \omega)\right)},
\end{aligned}
$$

and the linear first-order boundary value problem

$$
\begin{gathered}
u^{\prime}(t)+\rho u(t)=0, \quad t \in[0, \omega] \\
u(0)-u(\omega)=1 .
\end{gathered}
$$

has a unique solution given by

$$
\Phi_{1}(t)=\frac{e^{-\rho t}}{1-e^{-\rho \omega}}
$$

By a direct calculation, we can verify that

$$
\begin{aligned}
\Phi(t)= & \int_{0}^{t} \Phi_{2}(t-s) \Phi_{1}(s) d s \\
& +\int_{t}^{\omega} \Phi_{2}(\omega+t-s) \Phi_{1}(s) d s
\end{aligned}
$$

is the unique solution of the linear third-order boundary value (8). When $0<M<(2 \pi / \sqrt{3} \omega)^{3}, 0<\rho<2 \pi / \sqrt{3} \omega$ and, by $(11), \Phi_{2}(t)>0$ on $[0, \omega]$. Since $\Phi_{1}(t)>0$ on $[0, \omega]$, from (14), we see that $\Phi(t)>0$ for every $t \in[0, \omega]$. 
Let $0<M<(2 \pi / \sqrt{3} \omega)^{3}$. Then, the solution of $(8) \Phi(t)>$ 0 for every $t \in[0, \omega]$. If $h \in C_{\omega}^{+}(\mathbb{R})$ and $h(t) \neq 0$, by $(9)$, the $\omega$-periodic solution $u=P h$ of (7) is positive. We will show that the $\omega$-periodic solution has stronger positivity. Let

$$
\begin{gathered}
\sigma=\frac{\min _{t \in I} \Phi(t)}{\max _{t \in I} \Phi(t)}, \quad C_{1}=\frac{\max _{t \in I}\left|\Phi^{\prime}(t)\right|}{\min _{t \in I} \Phi(t)}, \\
C_{2}=\frac{\max _{t \in I}\left|\Phi^{\prime \prime}(t)\right|}{\min _{t \in I} \Phi(t)},
\end{gathered}
$$

where $I=[0, \omega]$. Choose a cone $K$ in $C_{\omega}^{2}(\mathbb{R})$ by

$$
\begin{aligned}
K=\left\{u \in C_{\omega}^{2}(\mathbb{R}) \cap C_{\omega}^{+}(\mathbb{R}) \mid u(t) \geq \sigma\|u\|_{C},\right. \\
\left.\quad\left|u^{\prime}(\tau)\right| \leq C_{1} u(t),\left|u^{\prime \prime}(\tau)\right| \leq C_{2} u(t), t, \tau \in \mathbb{R}\right\} .
\end{aligned}
$$

We have the following lemma.

Lemma 3. Let $0<M<(2 \pi / \sqrt{3} \omega)^{3}$. Then, for every $h \in$ $C_{\omega}^{+}(\mathbb{R})$, the $\omega$-periodic solution of $(7) u=P h \in K$.

Proof. Let $h \in C_{\omega}^{+}(\mathbb{R})$ and let $u=P h$. For every $t \in \mathbb{R}$, from (9), it follows that

$$
\begin{aligned}
u(t)=\int_{t-\omega}^{t} \Phi(t-s) h(s) d s & \leq \max _{r \in I} \Phi(r) \int_{t-\omega}^{t} h(s) d s \\
& =\max _{r \in I} \Phi(r) \int_{0}^{\omega} h(s) d s
\end{aligned}
$$

and, therefore,

$$
\|u\|_{C} \leq \max _{r \in I} \Phi(r) \int_{0}^{\omega} h(s) d s .
$$

Using (9) again, we obtain that

$$
\begin{aligned}
u(t) & =\int_{t-\omega}^{t} \Phi(t-s) h(s) d s \geq \min _{r \in I} \Phi(r) \int_{t-\omega}^{t} h(s) d s \\
& =\min _{r \in I} \Phi(r) \int_{0}^{\omega} h(s) d s \geq \sigma\|u\|_{C} .
\end{aligned}
$$

For every $\tau \in \mathbb{R}$, since

$$
u^{(i)}(\tau)=\int_{t-\omega}^{t} \Phi^{(i)}(\tau-s) h(s) d s, \quad i=1,2,
$$

we have

$$
\begin{aligned}
\left|u^{(i)}(\tau)\right| & \leq \int_{\tau-\omega}^{\tau}\left|\Phi^{(i)}(\tau-s)\right| h(s) d s \\
& \leq \max _{r \in I}\left|\Phi^{(i)}(r)\right| \int_{\tau-\omega}^{\tau} h(s) d s \\
& =\max _{r \in I}\left|\Phi^{(i)}(r)\right| \int_{0}^{\omega} h(s) d s \\
& =C_{i} \min _{r \in I} \Phi(r) \int_{0}^{\omega} h(s) d s \\
& \leq C_{i} u(t), \quad i=1,2 .
\end{aligned}
$$

Hence, $u \in K$.
Now, we consider the periodic solution problem of the linear third-order differential equation with variable coefficient

$$
u^{\prime \prime \prime}(t)+a(t) u(t)=h(t), \quad t \in \mathbb{R} .
$$

Let $a \in C_{\omega}(\mathbb{R})$ be a positive $\omega$-periodic function and satisfy the assumption

$$
\text { (H0) } 0<a(t)<\left(\frac{2 \pi}{\sqrt{3} \omega}\right)^{3} \text { for } t \in[0, \omega] \text {, }
$$

and set

$$
m=\min _{0 \leq t \leq \omega} a(t), \quad M=\max _{0 \leq t \leq \omega} a(t) .
$$

Then, $0<m \leq M<(2 \pi / \sqrt{3} \omega)^{3}$, and the conclusion of Lemma 3 holds. For (22), we have the following lemma:

Lemma 4. Let $a \in C_{\omega}(\mathbb{R})$ satisfy the assumption $(\mathrm{H} 0)$. Then, for every $h \in C_{\omega}(\mathbb{R})$, the linear equation (22) has a unique $\omega$ periodic solution $u:=$ Sh. Moreover, $S: C_{\omega}(\mathbb{R}) \rightarrow C_{\omega}^{2}(\mathbb{R})$ is a completely continuous linear operator and $S\left(C_{\omega}^{+}(\mathbb{R})\right) \subset K$.

Proof. Let $M$ and $m$ be the positive constants defined by (24) and let $P: C_{\omega}(\mathbb{R}) \rightarrow C_{\omega}(\mathbb{R})$ be the $\omega$-periodic solution operator of (7) given by (9). By Lemma $3, P\left(C_{\omega}^{+}(\mathbb{R})\right) \subset C_{\omega}^{+}(\mathbb{R})$, and $P: C_{\omega}(\mathbb{R}) \rightarrow C_{\omega}(\mathbb{R})$ is a positive linear bounded operator. We rewrite (22) into the form of

$$
u^{\prime \prime \prime}(t)+M u(t)=(M-a(t)) u(t)+h(t), \quad t \in \mathbb{R} .
$$

Then, it is easy to see that the $\omega$-periodic solution problem of (22) is equivalent to the operator equation in Banach space $C_{\omega}(\mathbb{R})$

$$
(I-P \circ B) u=P h,
$$

where $I$ is the identity operator in $C_{\omega}(\mathbb{R})$ and $B: C_{\omega}(\mathbb{R}) \rightarrow$ $C_{\omega}(\mathbb{R})$ is the product operator defined by

$$
B u(t)=(M-a(t)) u(t), \quad u \in C_{\omega}(\mathbb{R}),
$$

which is a positive linear bounded operator. We prove that the norm of $P \circ B$ in $\mathscr{L}\left(C_{\omega}(\mathbb{R}), C_{\omega}(\mathbb{R})\right)$ satisfies $\|P \circ B\|<1$.

For every $u \in C_{\omega}(\mathbb{R})$ and $t \in \mathbb{R}$, by definition (9) of $P$ and the positivity of $\Phi$, we have

$$
\begin{aligned}
|(P \circ B) u(t)| & =|P(B u)(t)| \\
& =\left|\int_{t-\omega}^{t} \Phi(t-s)(M-a(s)) u(s) d s\right| \\
& \leq \int_{t-\omega}^{t} \Phi(t-s)|(M-a(s)) u(s)| d s \\
& \leq(M-m)\|u\|_{C} \int_{t-\omega}^{t} \Phi(t-s) d s \\
& =(M-m)\|u\|_{C} \int_{0}^{\omega} \Phi(s) d s \\
& =\left(1-\frac{m}{M}\right)\|u\|_{C} .
\end{aligned}
$$


Therefore, $\|(P \circ B) u\|_{C} \leq(1-(m / M))\|u\|_{C}$. By the arbitrariness of $u \in C_{\omega}(\mathbb{R})$, we have $\|P \circ B\| \leq 1-(m / M)<1$.

Thus, $I-P \circ B$ has a bounded inverse operator given by the series

$$
(I-P \circ B)^{-1}=\sum_{n=0}^{\infty}(P \circ B)^{n} .
$$

Consequently, (26), equivalently (22), has a unique $\omega$ periodic solution

$$
u=(I-P \circ B)^{-1}(P h):=S h,
$$

where

$$
S=(I-P \circ B)^{-1} \circ P .
$$

By this and (29), we have

$$
\begin{aligned}
S & =(I-P \circ B)^{-1} \circ P=\sum_{n=0}^{\infty}(P \circ B)^{n} P \\
& =P+\sum_{n=1}^{\infty}(P \circ B)(P \circ B)^{n-1} P \\
& =P\left(I+\sum_{n=1}^{\infty} B(P \circ B)^{n-1} P\right) .
\end{aligned}
$$

Hence, $S$ can be expressed in the form of

$$
S=P \circ Q
$$

where

$$
Q=I+\sum_{n=1}^{\infty} B(P \circ B)^{n-1} P,
$$

which is a linear bounded operator from $C_{\omega}(\mathbb{R})$ into $C_{\omega}(\mathbb{R})$. By Lemma $1, P: C_{\omega}(\mathbb{R}) \rightarrow C^{2}(\mathbb{R})$ is completely continuous. Thus, from (33), we see that $S: C_{\omega}(\mathbb{R}) \rightarrow C_{\omega}^{2}(\mathbb{R})$ is a completely continuous linear operator.

By the positivity of $P$ and $B$, from the expression (34) of $Q$, we see that $Q: C_{\omega}(\mathbb{R}) \rightarrow C_{\omega}(\mathbb{R})$ is a positive linear operator. Hence, for every $h \in C_{\omega}^{+}(\mathbb{R}), h_{1}=Q h \in C_{\omega}^{+}(\mathbb{R})$. By (33) and Lemma 3, $u=S h=P(Q h)=P h_{1} \in K$. Thus, $S\left(C_{\omega}^{+}(\mathbb{R})\right) \subset K$.

The proof of Lemma 4 is completed.

Let $f: \mathbb{R} \times[0, \infty) \times \mathbb{R}^{2} \rightarrow[0, \infty)$ be a continuous function. For every $u \in K$, set

$$
\begin{array}{r}
F(u)(t):=f\left(t, u\left(t-\tau_{0}\right), u^{\prime}\left(t-\tau_{1}\right), u^{\prime \prime}\left(t-\tau_{2}\right)\right) \\
t \in \mathbb{R} .
\end{array}
$$

Then, $F: K \rightarrow C_{\omega}^{+}(\mathbb{R})$ is continuous. Now, we define a mapping $A: K \rightarrow C_{\omega}^{2}(\mathbb{R})$ by

$$
A=S \circ F,
$$

where $S: C_{\omega}(\mathbb{R}) \rightarrow C_{\omega}^{2}(\mathbb{R})$ is the periodic solution operator of (22). By Lemma 4, we have the following lemma.
Lemma 5. Let $a \in C_{\omega}(\mathbb{R})$ satisfy the assumption ( $\left.\mathrm{H} 0\right)$. Then, the operator $A: K \rightarrow K$ defined by (36) is completely continuous.

By the definition of operator $S$ and Lemma 4, the positive $\omega$-periodic solution of (1) is equivalent to the nonzero fixed point of $A$. We will find the nonzero fixed point of $A$ by using the fixed-point index theory in cones.

We recall some concepts and conclusions on the fixedpoint index in $[15,16]$. Let $E$ be a Banach space and $K \subset E$ be a closed convex cone in $E$. Assume $\Omega$ is a bounded open subset of $E$ with boundary $\partial \Omega$ and $K \cap \Omega \neq \emptyset$. Let $A: K \cap$ $\bar{\Omega} \rightarrow K$ be a completely continuous mapping. If $A u \neq u$ for any $u \in K \cap \partial \Omega$, then the fixed-point index $i(A, K \cap \Omega, K)$ has definition. One important fact is that if $i(A, K \cap \Omega, K) \neq 0$, then $A$ has a fixed point in $K \cap \Omega$. The following two lemmas in $[24,25]$ are needed in our argument.

Lemma 6. Let $\Omega$ be a bounded open subset of $E$ with $\theta \in \Omega$, and let $A: K \cap \bar{\Omega} \rightarrow K$ be a completely continuous mapping. If $\lambda A u \neq u$ for every $u \in K \cap \partial \Omega$ and $0<\lambda \leq 1$, then $i(A, K \cap$ $\Omega, K)=1$.

Lemma 7. Let $\Omega$ be a bounded open subset of $E$ and let $A$ : $K \cap \bar{\Omega} \rightarrow K$ be a completely continuous mapping. If there exists an $\in K \backslash\{\theta\}$ such that $u-A u \neq \mu$ for every $u \in K \cap \partial \Omega$ and $\mu \geq 0$, then $i(A, K \cap \Omega, K)=0$.

In next section, we will use Lemma 6 and Lemma 7 to discuss the existence of positive $\omega$-periodic solutions of (1).

\section{Main Results}

We consider the the existence of positive $\omega$-periodic solutions of the third-order delay equation (1). Let $a \in C_{\omega}(\mathbb{R})$ satisfy the assumption ( $\mathrm{H} 0)$ and let $M, m$ be the positive constants defined by (24). Let $f \in C\left(\mathbb{R} \times[0, \infty) \times \mathbb{R}^{2},[0, \infty)\right)$, and $f(t, x, y, z)$ be $\omega$-periodic in $t$. Let $C_{1}$ and $C_{2}$ be the constants defined by (15) and let $I=[0, \omega]$. To be convenient, we introduce the notations

$$
\begin{aligned}
& f_{0}=\liminf _{x \rightarrow 0^{+}} \min _{t \in I,|y| \leq C_{1} x,|z| \leq C_{2} x} \frac{f(t, x, y, z)}{x}, \\
& f^{0}=\limsup _{x \rightarrow 0^{+}} \max _{t \in I,|y| \leq C_{1} x,|z| \leq C_{2} x} \frac{f(t, x, y, z)}{x}, \\
& f_{\infty}=\liminf _{x \rightarrow+\infty} \min _{t \in I,|y| \leq C_{1} x,|z| \leq C_{2} x} \frac{f(t, x, y, z)}{x}, \\
& f^{\infty}=\limsup _{x \rightarrow+\infty} \max _{t \in I,|y| \leq C_{1} x,|z| \leq C_{2} x} \frac{f(t, x, y, z)}{x} .
\end{aligned}
$$

Our main results are as follows.

Theorem 8. Let $a \in C_{\omega}(\mathbb{R})$ satisfy the assumption $(\mathrm{H} 0)$, let $f: \mathbb{R} \times[0, \infty) \times \mathbb{R}^{2} \rightarrow \mathbb{R}$ be continuous, and let $f(t, x, y, z)$ be $\omega$-periodic in $t$. If $f$ satisfies the condition

$$
\text { (H1) } f^{0}<m, \quad f_{\infty}>M,
$$

then (1) has at least one positive $\omega$-periodic solution. 
Theorem 9. Let $a \in C_{\omega}(\mathbb{R})$ satisfy the assumption (H0), let $f: \mathbb{R} \times[0, \infty) \times \mathbb{R}^{2} \rightarrow \mathbb{R}$ be continuous, and let $f(t, x, y, z)$ be $\omega$-periodic in $t$. If $f$ satisfies the condition

$$
\text { (H2) } f_{0}>M, \quad f^{\infty}<m,
$$

then (1) has at least one positive $\omega$-periodic solution.

In Theorem 8 , the condition (H1) allows that $f(t, x, y, z)$ is superlinear growth on $x, y$, and $z$. For the application, see Example 10. In Theorem 9, the condition (H2) allows $f(t, x, y, z)$ sublinear growth on $x, y$, and $z$. See Example 11 .

Proof of Theorem 8. Choose working space $E=C_{\omega}^{2}(\mathbb{R})$. Let $K \subset C_{\omega}^{2}(\mathbb{R})$ be the cone in $C_{\omega}^{2}(\mathbb{R})$ defined by (16) and let $A: K \rightarrow K$ be the completely continuous operator defined by (36). Then, the positive $\omega$-periodic solution of (1) is equivalent to nontrivial fixed point of $A$. Let $0<r<R<$ $+\infty$ and set

$$
\begin{aligned}
& \Omega_{1}=\left\{u \in C_{\omega}^{2}(\mathbb{R}) \mid\|u\|_{C^{2}}<r\right\}, \\
& \Omega_{2}=\left\{u \in C_{\omega}^{2}(\mathbb{R}) \mid\|u\|_{C^{2}}<R\right\} .
\end{aligned}
$$

We show that the operator $A$ has a fixed point in $K \cap\left(\Omega_{2} \backslash \bar{\Omega}_{1}\right)$ when $r$ is small enough and $R$ is large enough.

By the assumption of $f^{0}<m$ and the definition of $f^{0}$, there exist $\varepsilon \in(0, m)$ and $\delta>0$, such that

$$
\begin{aligned}
& f(t, x, y, z) \leq(m-\varepsilon) x, \quad t \in I, \\
& |y| \leq C_{1} x, \quad|z| \leq C_{2} x, \quad 0<x \leq \delta .
\end{aligned}
$$

Let $r \in(0, \delta)$. We now prove that $A$ satisfies the condition of Lemma 6 in $K \cap \partial \Omega_{1}$; namely, $\lambda A u \neq u$ for every $u \in K \cap \partial \Omega_{1}$ and $0<\lambda \leq 1$. In fact, if there exist $u_{0} \in K \cap \partial \Omega_{1}$ and $0<\lambda_{0} \leq 1$ such that $\lambda_{0} A u_{0}=u_{0}$, since $u_{0}=S\left(\lambda_{0}\left(u_{0}\right)\right)$, by Lemma 4 and the definition of $S$ and $F, u_{0} \in C_{\omega}^{3}(\mathbb{R})$ satisfies the delay differential equation

$$
\begin{aligned}
& u_{0}^{\prime \prime \prime}(t)+a(t) u_{0}(t) \\
&=\lambda_{0} f_{1}\left(t, u_{0}\left(t-\tau_{0}\right), u_{0}^{\prime}\left(t-\tau_{1}\right), u_{0}^{\prime \prime}\left(t-\tau_{2}\right)\right), \\
& t \in \mathbb{R} .
\end{aligned}
$$

Since $u_{0} \in K \cap \partial \Omega_{1}$, by the definitions of $K$ and $\Omega_{1}$, we have

$$
\begin{gathered}
\left|u_{0}^{\prime}\left(t-\tau_{1}\right)\right| \leq C_{1} u_{0}\left(t-\tau_{0}\right), \\
\left|u_{0}^{\prime \prime}\left(t-\tau_{2}\right)\right| \leq C_{2} u_{0}\left(t-\tau_{0}\right), \\
0<\sigma\left\|u_{0}\right\|_{C} \leq u_{0}\left(t-\tau_{0}\right) \\
\leq\left\|u_{0}\right\|_{C^{2}}=r<\delta, \quad t \in \mathbb{R} .
\end{gathered}
$$

Hence, from (41), it follows that

$$
\begin{aligned}
& f_{1}\left(t, u_{0}\left(t-\tau_{0}\right), u_{0}^{\prime}\left(t-\tau_{1}\right), u_{0}^{\prime \prime}\left(t-\tau_{2}\right)\right) \\
& \quad \leq(m-\varepsilon) u_{0}\left(t-\tau_{0}\right), \quad t \in \mathbb{R} .
\end{aligned}
$$

By this inequality and (42), we have

$$
\begin{aligned}
& u_{0}^{\prime \prime \prime}(t)+a(t) u_{0}(t) \leq \lambda_{0}(m-\varepsilon) u_{0}\left(t-\tau_{0}\right) \\
& \quad \leq(m-\varepsilon) u_{0}\left(t-\tau_{0}\right), \quad t \in \mathbb{R} .
\end{aligned}
$$

Integrating both sides of this inequality from 0 to $\omega$ and using the periodicity of $u_{0}(t)$, we have

$$
\begin{aligned}
\int_{0}^{\omega} a(t) u_{0}(t) d t & \leq(m-\varepsilon) \int_{0}^{\omega} u_{0}\left(t-\tau_{0}\right) d t \\
& =(m-\varepsilon) \int_{0}^{\omega} u_{0}(t) d t .
\end{aligned}
$$

Hence, we obtain that

$$
m \int_{0}^{\omega} u_{0}(t) d t \leq \int_{0}^{\omega} a(t) u_{0}(t) d t \leq(m-\varepsilon) \int_{0}^{\omega} u_{0}(t) d s .
$$

Since $\int_{0}^{\omega} u_{0}(t) d t \geq \omega \sigma\left\|u_{0}\right\|_{C}>0$, from (47), it follows that $m \leq m-\varepsilon$, which is a contradiction. Hence, $A$ satisfies the condition of Lemma 6 in $K \cap \partial \Omega_{1}$. By Lemma 6 , we have

$$
i\left(A, K \cap \Omega_{1}, K\right)=1 .
$$

On the other hand, since $f_{\infty}>M$, by the definition of $f_{\infty}$, there exist $\varepsilon_{1}>0$ and $H>0$ such that

$$
\begin{aligned}
& f(t, x, y, z) \geq\left(M+\varepsilon_{1}\right) x, \quad t \in I, \\
& |y| \leq C_{1} x, \quad|z| \leq C_{1} x, \quad x \geq H .
\end{aligned}
$$

Choose $R>\max \left\{\left(\left(1+C_{1}+C_{2}\right) / \sigma\right) H, \delta\right\}$ and $e(t) \equiv 1$. Clearly, $e \in K \backslash\{\theta\}$. We show that $A$ satisfies the condition of Lemma 7 in $K \cap \partial \Omega_{2}$; namely, $u-A u \neq \mu e$ for every $u \in K \cap \partial \Omega_{2}$ and $\mu \geq 0$. In fact, if there exist $u_{1} \in K \cap \partial \Omega_{2}$ and $\mu_{1} \geq 0$ such that $u_{1}-A u_{1}=\mu_{1} e$, since $u_{1}-\mu_{1} e=S\left(F\left(u_{1}\right)\right)$, by Lemma 4 and the definition of $S$ and $F, u_{1} \in C_{\omega}^{3}(\mathbb{R})$ satisfies the differential equation

$$
\begin{aligned}
& u_{1}^{\prime \prime \prime}(t)+a(t)\left(u_{1}(t)-\mu_{1}\right) \\
&=f_{1}\left(t, u_{1}\left(t-\tau_{0}\right), u_{1}^{\prime}\left(t-\tau_{1}\right), u_{1}^{\prime \prime}\left(t-\tau_{2}\right)\right), \\
& t \in \mathbb{R} .
\end{aligned}
$$

Since $u_{1} \in K \cap \partial \Omega_{2}$, by the definition of $K$ and $\Omega_{2}$, we have

$$
\begin{gathered}
u_{1}(t) \geq \sigma\left\|u_{1}\right\|_{C^{\prime}} \\
\left|u_{1}^{\prime}(r)\right| \leq C_{1} u_{1}(t), \quad\left|u_{1}^{\prime \prime}(r)\right| \leq C_{2} u_{1}(t), \quad \forall r, t \in \mathbb{R} .
\end{gathered}
$$

By the latter inequalities of (51), we have

$$
\left\|u_{1}^{\prime}\right\|_{C} \leq C_{1}\left\|u_{1}\right\|_{C}, \quad\left\|u_{1}^{\prime \prime}\right\|_{C} \leq C_{2}\left\|u_{1}\right\|_{C} .
$$

These inequalities mean that

$$
\left\|u_{1}\right\|_{C^{2}}=\left\|u_{1}\right\|_{C}+\left\|u_{1}^{\prime}\right\|_{C}+\left\|u_{1}^{\prime \prime}\right\|_{C} \leq\left(1+C_{1}+C_{2}\right)\left\|u_{1}\right\|_{C^{*}}
$$


Hence, we obtain that

$$
\left\|u_{1}\right\|_{C} \geq \frac{1}{1+C_{1}+C_{2}}\left\|u_{1}\right\|_{C^{2}} .
$$

By (54) and the former inequality of (51), we have

$$
u_{1}(t) \geq \sigma\left\|u_{1}\right\|_{C} \geq \frac{\sigma}{1+C_{1}+C_{2}}\left\|u_{1}\right\|_{C^{2}}=\frac{\sigma R}{1+C_{1}+C_{2}}>H
$$

From this, the latter inequalities of (51) and (49), it follows that

$$
\begin{gathered}
f_{1}\left(t, u_{1}\left(t-\tau_{0}\right), u_{1}^{\prime}\left(t-\tau_{1}\right), u_{1}^{\prime \prime}\left(t-\tau_{2}\right)\right) \\
\geq\left(M+\varepsilon_{1}\right) u_{1}\left(t-\tau_{0}\right), \quad t \in I .
\end{gathered}
$$

By this inequality and (50), we have

$$
u_{1}^{\prime \prime \prime}(t)+a(t)\left(u_{1}(t)-\mu_{1}\right) \geq\left(M+\varepsilon_{1}\right) u_{1}\left(t-\tau_{0}\right), \quad t \in I .
$$

Integrating this inequality on $I$ and using the periodicity of $u_{1}$, we have

$$
\begin{aligned}
\int_{0}^{\omega} a(t) u_{1}(t) d t-\mu_{1} \omega & \geq\left(M+\varepsilon_{1}\right) \int_{0}^{\omega} u_{1}\left(t-\tau_{0}\right) d t \\
& =\left(M+\varepsilon_{1}\right) \int_{0}^{\omega} u_{1}(t) d t .
\end{aligned}
$$

Hence, we obtain that

$$
\begin{aligned}
M \int_{0}^{\omega} u_{1}(t) d t & \geq \int_{0}^{\omega} a(t) u_{1}(t) d t-\mu_{1} \omega \\
& \geq\left(M+\varepsilon_{1}\right) \int_{0}^{\omega} u_{1}(t) d t
\end{aligned}
$$

Since $\int_{0}^{\omega} u_{1}(t) d t \geq \omega \sigma\left\|u_{1}\right\|_{C}>0$, from (59), it follows that $M \geq M+\varepsilon_{1}$, which is a contradiction. This means that $A$ satisfies the condition of Lemma 7 in $K \cap \partial \Omega_{2}$. By Lemma 7,

$$
i\left(A, K \cap \Omega_{2}, K\right)=0 .
$$

Now, by the additivity of fixed-point index, (48), and (60), we have

$$
\begin{aligned}
i\left(A, K \cap\left(\Omega_{2} \backslash \bar{\Omega}_{1}\right), K\right)= & i\left(A, K \cap \Omega_{2}, K\right) \\
& -i\left(A, K \cap \Omega_{1}, K\right)=-1 .
\end{aligned}
$$

Hence, $A$ has a fixed point in $K \cap\left(\Omega_{2} \backslash \bar{\Omega}_{1}\right)$, which is a positive $\omega$-periodic solution of (1).

Proof of Theorem 9. Let $\Omega_{1}, \Omega_{2} \subset C_{\omega}^{2}(\mathbb{R})$ be defined by $(40)$. We prove that the operator $A$ defined by (36) has a fixed point in $K \cap\left(\Omega_{2} \backslash \bar{\Omega}_{1}\right)$ if $r$ is small enough and $R$ is large enough.
By the assumption of $f_{0}>M$ and the definition of $f_{0}$, there exist $\varepsilon>0$ and $\delta>0$, such that

$$
\begin{aligned}
& f(t, x, y, z) \geq(M+\varepsilon) x, \quad t \in I, \\
& |y| \leq C_{1} x, \quad|z| \leq C_{2} x, \quad 0<x \leq \delta .
\end{aligned}
$$

Let $r \in(0, \delta)$ and let $e(t) \equiv 1$. We prove that $A$ satisfies the condition of Lemma 7 in $K \cap \partial \Omega_{1}$; namely, $u-A u \neq \mu e$ for every $u \in K \cap \partial \Omega_{1}$ and $\mu \geq 0$. In fact, if there exist $u_{0} \in$ $K \cap \partial \Omega_{1}$ and $\mu_{0} \geq 0$ such that $u_{0}-A u_{0}=\mu_{0} e$, since $u_{0}-\mu_{0} e=$ $S\left(F\left(u_{0}\right)\right)$, by Lemma 4 and the definition of $S$ and $F, u_{0}(t) \epsilon$ $C_{\omega}^{3}(\mathbb{R})$ satisfies the differential equation

$$
\begin{array}{r}
u_{0}^{\prime \prime \prime}(t)+a(t)\left(u_{0}(t)-\mu_{0}\right) \\
=f_{1}\left(t, u_{0}\left(t-\tau_{0}\right), u_{0}^{\prime}\left(t-\tau_{1}\right), u_{0}^{\prime \prime}\left(t-\tau_{2}\right)\right), \\
t \in \mathbb{R} .
\end{array}
$$

Since $u_{0} \in K \cap \partial \Omega_{1}$, by the definitions of $K$ and $\Omega_{1}, u_{0}$ satisfies (43). From (43) and (62), we see that

$$
\begin{aligned}
& f_{1}\left(t, u_{0}\left(t-\tau_{0}\right), u_{0}^{\prime}\left(t-\tau_{1}\right), u_{0}^{\prime \prime}\left(t-\tau_{2}\right)\right) \\
& \geq(M+\varepsilon) u_{0}\left(t-\tau_{0}\right), \quad t \in \mathbb{R} .
\end{aligned}
$$

From this and (63), it follows that

$$
u_{0}^{\prime \prime \prime}(t)+a(t)\left(u_{0}(t)-\mu_{0}\right) \geq(M+\varepsilon) u_{0}\left(t-\tau_{0}\right), \quad t \in \mathbb{R} .
$$

Integrating this inequality on $I$ and using the periodicity of $u_{0}(t)$, we have

$$
\begin{aligned}
\int_{0}^{\omega} a(t) u_{0}(t) d t-\mu_{0} \omega & \geq(M+\varepsilon) \int_{0}^{\omega} u_{0}\left(t-\tau_{0}\right) d t \\
& =(M+\varepsilon) \int_{0}^{\omega} u_{0}(t) d t .
\end{aligned}
$$

Consequently,

$$
\begin{aligned}
M \int_{0}^{\omega} u_{0}(t) d t & \geq \int_{0}^{\omega} a(t) u_{0}(t) d t-\mu_{0} \omega \\
& \geq(M+\varepsilon) \int_{0}^{\omega} u_{0}(t) d t .
\end{aligned}
$$

Since $\int_{0}^{\omega} u_{0}(t) d t \geq \omega \sigma\left\|u_{0}\right\|_{C}>0$, from (67), it follows that $M \geq M+\varepsilon$, which is a contradiction. Hence, $A$ satisfies the condition of Lemma 7 in $K \cap \partial \Omega_{1}$. By Lemma 7, we have

$$
i\left(A, K \cap \Omega_{1}, K\right)=0 .
$$

Since $f^{\infty}<m$, by the definition of $f^{\infty}$, there exist $\varepsilon_{1} \in$ $(0, m)$ and $H>0$ such that

$$
\begin{aligned}
& f(t, x, y, z) \leq\left(m-\varepsilon_{1}\right) x, \quad t \in I, \\
& |y| \leq C_{1} x, \quad|z| \leq C_{2} x, \quad x \geq H .
\end{aligned}
$$


Choosing $R>\max \left\{\left(\left(1+C_{1}+C_{2}\right) / \sigma\right) H, \delta\right\}$, we show that $A$ satisfies the condition of Lemma 6 in $K \cap \partial \Omega_{2}$; namely, $\lambda A u \neq u$ for every $u \in K \cap \partial \Omega_{2}$ and $0<\lambda \leq 1$. In fact, if there exist $u_{1} \in K \cap \partial \Omega_{2}$ and $0<\lambda_{1} \leq 1$ such that $\lambda_{1} A u_{1}=u_{1}$, since $u_{1}=S\left(\lambda_{1}\left(F\left(u_{1}\right)\right)\right)$, by Lemma 4 and the definition of $S$ and $F, u_{1} \in C_{\omega}^{3}(\Omega)$ satisfies the differential equation

$$
\begin{aligned}
& u_{1}^{\prime \prime}(t)+a(t) u_{1}(t) \\
& \quad=\lambda_{1} f_{1}\left(t, u_{1}\left(t-\tau_{0}\right), u_{1}^{\prime}\left(t-\tau_{1}\right), u_{1}^{\prime \prime}\left(t-\tau_{2}\right)\right), \quad t \in \mathbb{R} .
\end{aligned}
$$

Since $u_{1} \in K \cap \partial \Omega_{2}$, by the definition of $K, u_{1}$ satisfies (51). By (51) we can show that $u_{1}$ satisfies (54). By (51) and (54), we have,

$$
\begin{aligned}
u_{1}(t) & \geq \sigma\left\|u_{1}\right\|_{C} \geq \frac{\sigma}{1+C_{1}+C_{2}}\left\|u_{1}\right\|_{C^{2}} \\
& =\frac{\sigma R}{1+C_{1}+C_{2}}>H, \quad t \in I .
\end{aligned}
$$

Since $u_{1}$ satisfies (51) and (71), from (69), it follows that

$$
\begin{aligned}
& f_{1}\left(t, u_{1}\left(t-\tau_{0}\right), u_{1}^{\prime}\left(t-\tau_{1}\right), u_{1}^{\prime \prime}\left(t-\tau_{2}\right)\right) \\
& \quad \leq\left(m-\varepsilon_{1}\right) u_{1}\left(t-\tau_{0}\right), \quad t \in I .
\end{aligned}
$$

By this and (70), we have

$$
\begin{aligned}
u_{1}^{\prime \prime \prime}(t)+a(t) u_{1}(t) & \leq \lambda_{1}\left(m-\varepsilon_{1}\right) u_{1}\left(t-\tau_{0}\right) \\
& \leq\left(m-\varepsilon_{1}\right) u_{1}\left(t-\tau_{0}\right), \quad t \in \mathbb{R} .
\end{aligned}
$$

Integrating this inequality on $I$ and using the periodicity of $u_{1}(t)$, we obtain that

$$
\begin{aligned}
\int_{0}^{\omega} a(t) u_{1}(t) d t & \leq\left(m-\varepsilon_{1}\right) \int_{0}^{\omega} u_{1}\left(t-\tau_{0}\right) d t \\
& =\left(m-\varepsilon_{1}\right) \int_{0}^{\omega} u_{1}(t) d t .
\end{aligned}
$$

Hence, we have

$$
m \int_{0}^{\omega} u_{1}(t) d t \leq \int_{0}^{\omega} a(t) u_{1}(t) d t \leq\left(m-\varepsilon_{1}\right) \int_{0}^{\omega} u_{1}(t) d t .
$$

Since $\int_{0}^{\omega} u_{1}(t) d t \geq \omega \sigma\left\|u_{1}\right\|_{C}>0$, from (75), it follows that $m \leq m-\varepsilon_{1}$, which is a contradiction. This means that $A$ satisfies the condition of Lemma 6 in $K \cap \partial \Omega_{2}$. By Lemma 6 ,

$$
i\left(A, K \cap \Omega_{2}, K\right)=1 .
$$

Now, from (68) and (76), it follows that

$$
\begin{aligned}
i\left(A, K \cap\left(\Omega_{2} \backslash \bar{\Omega}_{1}\right), K\right)= & i\left(A, K \cap \Omega_{2}, K\right) \\
& -i\left(A, K \cap \Omega_{1}, K\right)=1 .
\end{aligned}
$$

Hence, $A$ has a fixed point in $K \cap\left(\Omega_{2} \backslash \bar{\Omega}_{1}\right)$, which is a positive $\omega$-periodic solution of (1).
Example 10. Consider the superlinear third-order delay differential equation

$$
\begin{aligned}
u^{\prime \prime \prime}(t)+a(t) u(t)= & b_{0}(t) u^{2}(t-\pi)+b_{1}(t)\left(u^{\prime}(t-\pi)\right)^{2} \\
& +b_{2}(t)\left(u^{\prime \prime}(t-\pi)\right)^{2}, \quad t \in \mathbb{R},
\end{aligned}
$$

where $a, b_{i} \in C_{2 \pi}(\mathbb{R}), i=0,1,2$, and satisfy the conditions

$$
0<a(t)<\frac{1}{(3 \sqrt{3})}, \quad b_{0}(t), b_{1}(t), b_{2}(t)>0, \quad t \in \mathbb{R} .
$$

It is easy to verify that $a(t)$ satisfies the assumption (H0) for $\omega=2 \pi$ and

$$
f(t, x, y, z)=b_{0}(t) x^{2}+b_{1}(t) y^{2}+b_{2}(t) z^{2}
$$

satisfies the assumption ( $\mathrm{H} 1)$ with $f^{0}=0$ and $f_{\infty}=$ $+\infty$. Hence, by Theorem $8,(78)$ has at least one positive $2 \pi$ periodic solution.

Example 11. Consider the third-order delay differential equation

$$
\begin{aligned}
u^{\prime \prime \prime}(t) & +\left(\frac{1}{6}-\frac{1}{7} \sin ^{2} t\right) u(t) \\
= & c_{0}(t) \sqrt{|u(t-\pi)|}+c_{1}(t) \sqrt{\left|u^{\prime}(t-\pi)\right|} \\
& +c_{2}(t) \sqrt{\left|u^{\prime \prime}(t-\pi)\right|}, \quad t \in \mathbb{R},
\end{aligned}
$$

where $c_{0}, c_{1}$, and $c_{2} \in C_{2 \pi}(\mathbb{R})$ are positive $2 \pi$-periodic functions. It is easy to verify that $a(t)=(1 / 6)-(1 / 7) \sin ^{2} t$ satisfies the assumption (H0) for $\omega=2 \pi$. Let

$$
f(t, x, y, z)=c_{0}(t) \sqrt{|x|}+c_{1}(t) \sqrt{|y|}+b_{2}(t) \sqrt{|z|} .
$$

Then, $f^{0}=+\infty$ and $f_{\infty}=0$. Hence, $f(t, x, y, z)$ satisfies the assumption (H2). By Theorem 9, (81) has at least one positive $2 \pi$-periodic solution.

\section{Conflict of Interests}

The authors declare that there is no conflict of interests regarding the publication of this paper.

\section{Acknowledgments}

The research is supported by NNSFs of China (11261053, 11061031) and the NSF of Gansu Province (1208RJZA129).

\section{References}

[1] H. I. Freedman and J. H. Wu, "Periodic solutions of singlespecies models with periodic delay," SIAM Journal on Mathematical Analysis, vol. 23, no. 3, pp. 689-701, 1992. 
[2] L. Hatvani and T. Krisztin, "On the existence of periodic solutions for linear inhomogeneous and quasilinear functionaldifferential equations," Journal of Differential Equations, vol. 97, no. 1, pp. 1-15, 1992.

[3] A. Wan, D. Jiang, and X. Xu, "A new existence theory for positive periodic solutions to functional differential equations," Computers \& Mathematics with Applications, vol. 47, no. 8-9, pp. 1257-1262, 2004.

[4] S. Kang and G. Zhang, "Existence of nontrivial periodic solutions for first order functional differential equations," Applied Mathematics Letters, vol. 18, no. 1, pp. 101-107, 2005.

[5] Y. Luo, W. Wang, and J. Shen, "Existence of positive periodic solutions for two kinds of neutral functional differential equations," Applied Mathematics Letters, vol. 21, no. 6, pp. 581-587, 2008.

[6] J.-W. Li and S. S. Cheng, "Periodic solutions of a second order forced sublinear differential equation with delay," Applied Mathematics Letters, vol. 18, no. 12, pp. 1373-1380, 2005.

[7] Y. Wang, H. Lian, and W. Ge, "Periodic solutions for a second order nonlinear functional differential equation," Applied Mathematics Letters, vol. 20, no. 1, pp. 110-115, 2007.

[8] J. Wu and Z. Wang, "Two periodic solutions of second-order neutral functional differential equations," Journal of Mathematical Analysis and Applications, vol. 329, no. 1, pp. 677-689, 2007.

[9] Y. Wu, "Existence nonexistence and multiplicity of periodic solutions for a kind of functional differential equation with parameter," Nonlinear Analysis. Theory, Methods \& Applications A, vol. 70, no. 1, pp. 433-443, 2009.

[10] C. Guo and Z. Guo, "Existence of multiple periodic solutions for a class of second-order delay differential equations," Nonlinear Analysis. Real World Applications, vol. 10, no. 5, pp. 3285-3297, 2009.

[11] W.-S. Cheung, J. Ren, and W. Han, "Positive periodic solution of second-order neutral functional differential equations," Nonlinear Analysis. Theory, Methods \& Applications A, vol. 71, no. 9, pp. 3948-3955, 2009.

[12] Y. Li, "Positive periodic solutions of second-order differential equations with delays," Abstract and Applied Analysis, vol. 2012, Article ID 829783, 13 pages, 2012.

[13] Z. Zhao and Y. Xu, "Solitary waves for Korteweg-de Vries equation with small delay," Journal of Mathematical Analysis and Applications, vol. 368, no. 1, pp. 43-53, 2010.

[14] A. R. Aftabizadeh, J.-M. Xu, and C. P. Gupta, "Periodic boundary value problems for third order ordinary differential equations," Nonlinear Analysis. Theory, Methods \& Applications $A$, vol. 14, no. 1, pp. 1-10, 1990.

[15] P. Omari and M. Trombetta, "Remarks on the lower and upper solutions method for second- and third-order periodic boundary value problems," Applied Mathematics and Computation, vol. 50 , no. 1, pp. 1-21, 1992.

[16] A. Cabada, "The method of lower and upper solutions for second, third, fourth, and higher order boundary value problems," Journal of Mathematical Analysis and Applications, vol. 185, no. 2, pp. 302-320, 1994.

[17] A. Cabada, "The method of lower and upper solutions for third-order periodic boundary value problems," Journal of Mathematical Analysis and Applications, vol. 195, no. 2, pp. 568589, 1995.

[18] B. Mehri and M. Niksirat, "On the existence of periodic solutions for the quasi-linear third-order differential equation," Journal of Mathematical Analysis and Applications, vol. 261, no. 1, pp. 159-167, 2001.
[19] L. Kong, S. Wang, and J. Wang, "Positive solution of a singular nonlinear third-order periodic boundary value problem," Journal of Computational and Applied Mathematics, vol. 132, no. 2, pp. 247-253, 2001.

[20] P. Amster, P. De Nápoli, and M. C. Mariani, "Periodic solutions of a resonant third-order equation," Nonlinear Analysis. Theory, Methods \& Applications A, vol. 60, no. 3, pp. 399-410, 2005.

[21] J. Chu and Z. Zhou, "Positive solutions for singular nonlinear third-order periodic boundary value problems," Nonlinear Analysis. Theory, Methods \& Applications A, vol. 64, no. 7, pp. 1528-1542, 2006.

[22] Y. Feng, "On the existence and multiplicity of positive periodic solutions of a nonlinear third-order equation," Applied Mathematics Letters, vol. 22, no. 8, pp. 1220-1224, 2009.

[23] Y. Li, "Positive periodic solutions for fully third-order ordinary differential equations," Computers \& Mathematics with Applications, vol. 59, no. 11, pp. 3464-3471, 2010.

[24] K. Deimling, Nonlinear Functional Analysis, Springer, New York, NY, USA, 1985.

[25] D. J. Guo and V. Lakshmikantham, Nonlinear Problems in Abstract Cones, Academic Press, Boston, Mass, USA, 1988. 


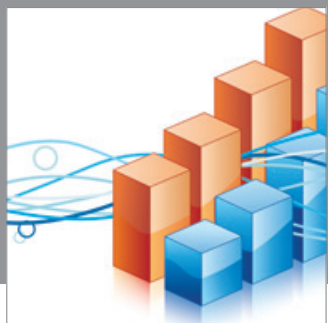

Advances in

Operations Research

mansans

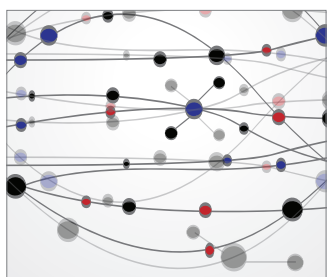

The Scientific World Journal
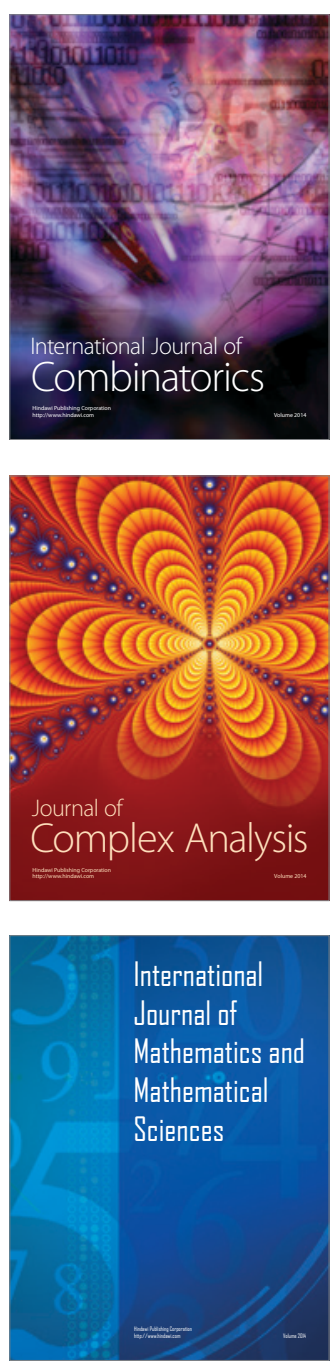
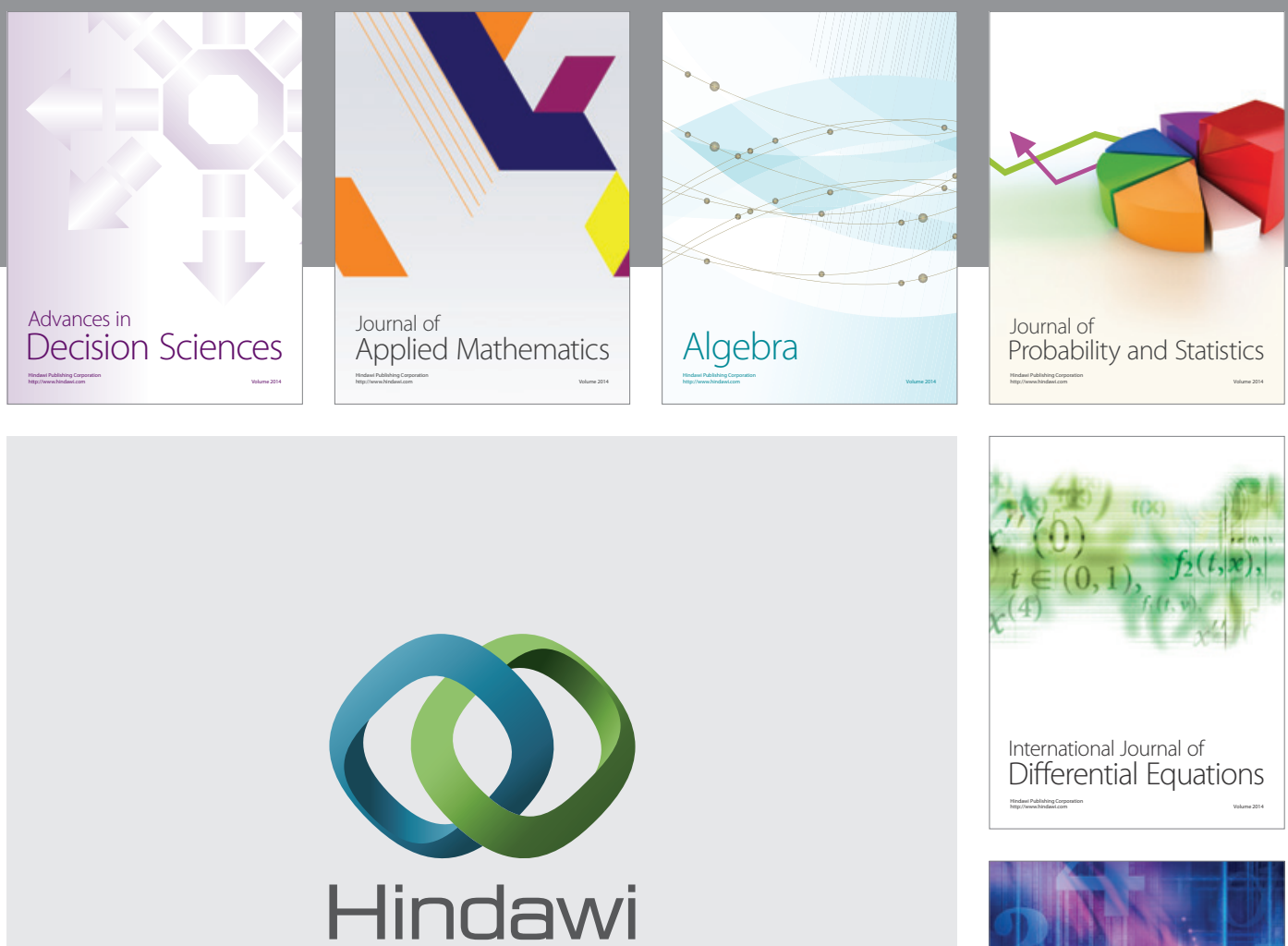

Submit your manuscripts at http://www.hindawi.com
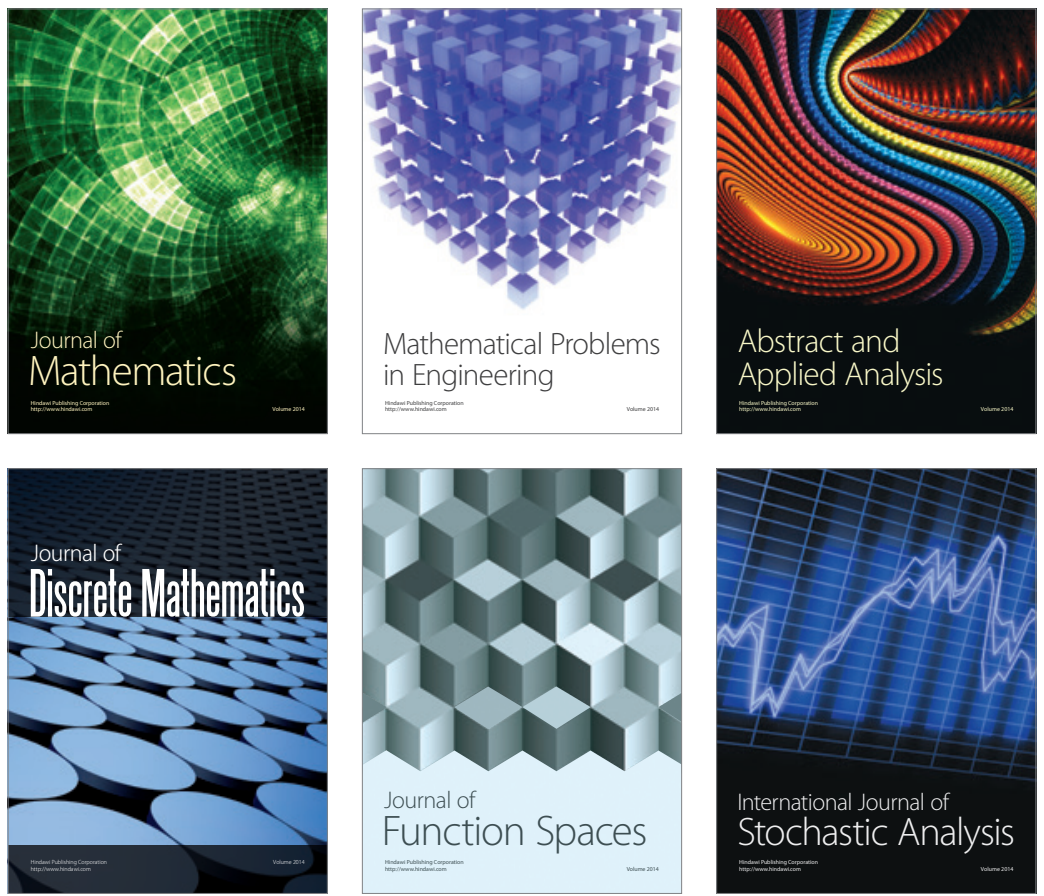

Journal of

Function Spaces

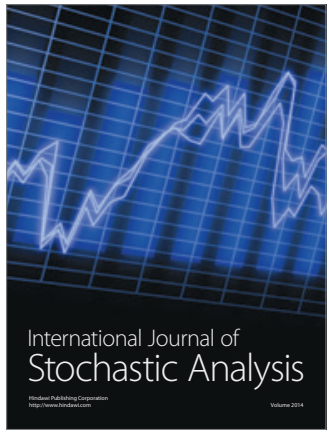

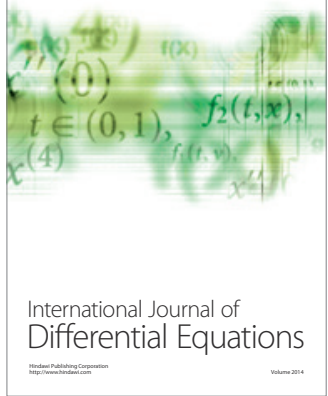
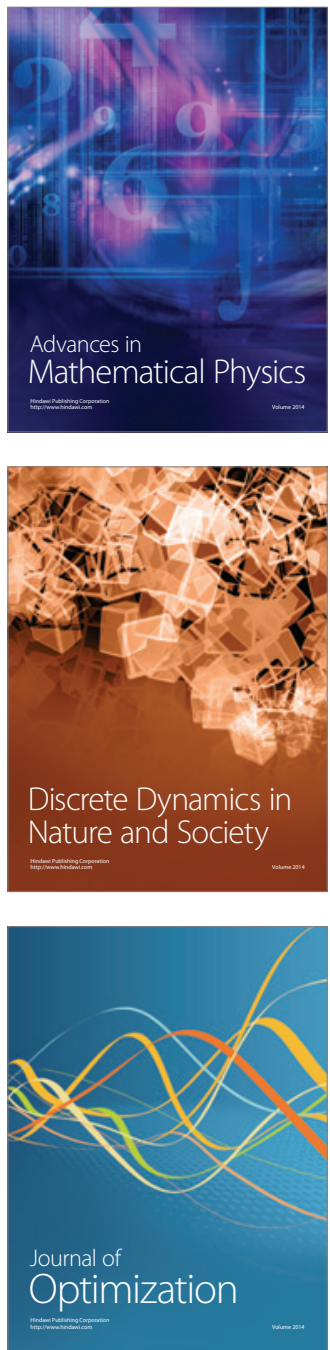